\title{
Presence of Sun Protection Factor (SPF) in the Tagetes Plants
}

\author{
Deepshikha Kushwaha ${ }^{1 *}$, Prashant Katiyar ${ }^{2}$, Yashodhara Verma ${ }^{3}$ \\ ${ }^{1}$ Research Scholar, Department of Biochemistry and Biochemical Engineering, SHUATS, Allahabad, India \\ ${ }^{2}$ Research Scholar, Department of Biochemistry and Biochemical Engineering, SHUATS, Allahabad, India \\ ${ }^{3}$ Assistant Professor, Department of Biochemistry and Biochemical Engineering, SHUATS, Allahabad, India
}

\begin{abstract}
*Address for Correspondence: Deepshikha Kushwaha, Research Scholar, Department of Biochemistry and Biochemical Engineering, Sam Higgin Bottom University of Agriculture, Technology and Sciences (SHUATS), Allahabad-211007, India E-mail: deep shikha200@yahoo.com
\end{abstract}

Received: 26 Jun 2021/ Revised: 23 Aug 2021/ Accepted: 03 Oct 2021

\begin{abstract}
Background: The marigold plant is widely used as herbal medicine due to presences of many phyto constituents. In ancient time extract of marigold flower used as the remedy against various skin problems.

Method: The aim of this study was determining the photo-protective activity of Tagetes Leaves and Flower by using UVSpectrophotometer. UV-Vis spectrophotometry measured the absorption rate of UV rays at different wavelength of each sample. To calculate the final sun protection factor (SPF) value, Mansur equation was appropriated method.

Results: The result of study demonstrates that the marigold plants having for 15 and 10 SPF for flowers and leaves respectively Conclusion: Marigold plant having natural SPF and it become a good source for formulation of bio-cosmetics.
\end{abstract}

Key-words: Photo-protective, Solar radiation, Sun Protection Factor, Tagetes plants, UV-Spectrophotometer

\section{INTRODUCTION}

Sunlight consists of a variety of wavelengths ranging from ultraviolet light to infrared and visible light. Direct skin exposure to solar radiation has some adverse effects. Excluding all sun rays, ultraviolet light has the more deleterious effects on the skin such as sunburns, skin ageing and over the long term exposure of UV light even causes skin cancer. These harmful radiations causes' deleterious effects in skin like sarcoma due to oxidative free radicals presence in an excessive amount and this amount raises continuously as the amount of UV-B radiation is constantly exposed ${ }^{[1]}$. The electromagnetic spectra of UV sun radiation can be further classified into three different regions: UVA, from 320 to $400 \mathrm{~nm}$; UVB, from 290 to $320 \mathrm{~nm}$ and UVC, from 200 to $290 \mathrm{~nm}{ }^{[2]}$. Usually when sunrays approach to earth atmosphere only UVB radiation partially filtered by

\section{How to cite this article}

Kushwaha D, Katiyar P, Verma Y. Presence of Sun Protection Factor (SPF) in the Tagetes Plants. SSR Inst. Int. J. Life Sci., 2021; 7(6): 2908-2912.

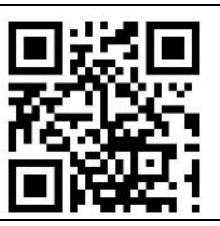

outer ozone layer and UV-A completely reaches to earth atmosphere while UV-C is completely absorbed at outer atmosphere. UV-A causes skin ageing, rashes in epidermal layer of skin, while UV-B having higher penetration power as compare to UV-A actually responsible for causing cancer in skin ${ }^{[3]}$.

Due to these facts, UV-B radiations exposure can be cut off by developing naturally synthesized cosmetics products, which has photo-protective action and provide nourishment to the skin tissues. This is possible owing to the presence of antioxidants, which restricts the formation of free radicals and help to reduce the chance of destructive skin effects of ultraviolet radiation. However, it is necessary to include such photo protective bioactive active compounds in the cosmetic formulation and measures their efficiency against UV-rays. The sunscreen efficacy is usually decided on the basis of Sun Protection Factor (SPF), defined as the UV energy required causing minimal erythema dose (MED) on protected skin to the UV energy required to produce a MED on unprotected skin ${ }^{[4]}$. The minimal erythemal dose (MED) is defined as the minimum dosage of UV light irradiation sufficient to produce a minimal, perceptible erythema on unprotected skins [5] for 
shortest time interval. It means higher the SPF value containing cosmetic products are beneficial and more effective against UV-B rays.

\section{SPF = Minimal erythema dose in sunscreen - protected skin / Minimal erythema dose in non sunscreen - protected skin}

Various synthetic products are available in the markets which are generally useful to protect skin from harmful UV rays. But these chemically synthesized products are usually not safe and effective ${ }^{[5-7]}$. This is big disadvantage of synthetic cosmetic products. Synthetic products are available in the market containing various harmful effects and not so much effective. To avoid this problem researcher's criteria were shifted towards the naturally synthesized products ${ }^{[8]}$. Many Researchers were focusing on naturally synthesized products, used as a cosmetic product like synthetic cosmetics. But the foremost criteria for naturally synthesized cosmetics are the presence of bioactive compounds ${ }^{[9]}$ generally found in natural resources and still some researchers were investigate to find out unknown and novel bioactive compounds, which has a protective action against harmful UV radiations. This can be possible by discovering new reaction mechanisms, which is helpful to search out these interesting bioactive compounds, and easier to study their structure function relationships in order to develop naturally synthesized photo protective active compounds and restrict an unwanted inhibitory product formation. Furthermore, the natural synthesized active compounds are already known and widely occurring, then it is possible to produce a high quality cosmetics product with significant SPF value characteristics available relatively at a low price ${ }^{[10,11]}$.

\section{MATERIALS AND METHODS}

Reagents and samples- Ethanol AR. Marigold plants were collected from the field of SHUATS, Allahabad.

Apparatus- Cistronic UV/Visible spectrophotometer 2202.

\section{Methods}

Sample preparation- Weighed (100 mg) sample ultrasonicated with $100 \mathrm{ml}$ analytical grade ethanol for 5 minutes. Sonicated samples were filtered. First $10 \mathrm{ml}$ sample was discarded, out of remained filtrated $5 \mathrm{ml}$ was relocated to volumetric flask and make up volume up to
$50 \mathrm{ml}$ with diluted ethanol. Then take $5 \mathrm{ml}$ sample and further 5 times diluted with ethanol. Prepared sample was read at the range from 290-320 nm to take absorption spectra at every $5 \mathrm{~nm}$ against ethanol blank in triplicate manner for 30 minutes. Obtained data was calculated by using Mansur equation mentioned below-

$$
\operatorname{SPF}(\text { spectrometry })=\operatorname{CF} \times \Sigma^{320}{ }_{290} \mathrm{EE}(\lambda) \times I(\lambda) \times \text { abs }(\lambda)
$$

Where $\mathrm{CF}=$ correction factor $(=10), \mathrm{EE}(\lambda)=$ Erythemal effect spectrum; $(\lambda)$; I $(\lambda)=$ intensity of the sunlight at a wavelength $(\lambda)$; abs $(\lambda)=$ sample absorbance at wavelength $(\lambda)$

The values of $\operatorname{EE}(\lambda)$ and $I(\lambda)$ were calculated as previously by Sayre et al. ${ }^{[12]}$ as described in Table 1.

Table 1: Erythemogenic effect relationship (EE) versus radiation intensity $(I)$ according to the wavelength $(\lambda)$

\begin{tabular}{cc}
\hline$\lambda(\mathrm{nm})$ & EE x I (normalized) \\
\hline 290 & 0.0150 \\
295 & 0.0817 \\
300 & 0.2874 \\
305 & 0.3278 \\
310 & 0.1864 \\
315 & 0.0839 \\
320 & 0.0180 \\
\hline
\end{tabular}

Statistical Analysis- The present data/results were analyzed the variance through a post hoc (ANOVA) multiple evaluation t-test $(p \leq 0.01)$ by using STAT PAC Version 14.

\section{RESULTS}

The SPF values for marigold leaves and flower were determined through the spectrophotometric method and calculated by Mansur equation. The results were represented in Fig. 1. A quantitative method to ensure the formulation of sunscreen is sun protection factor. It is efficient to avoid and heal skin against sunburn and different other skin damages. SPF containing cosmetics products absorbed or reflected a wide range UV rays in between 290-320 $\mathrm{nm}$. Graphically it shows the sun protection factor (SPF) was found at maximum at $305 \mathrm{~nm}$ and minimum at $290 \mathrm{~nm}$ (Fig. 2). 


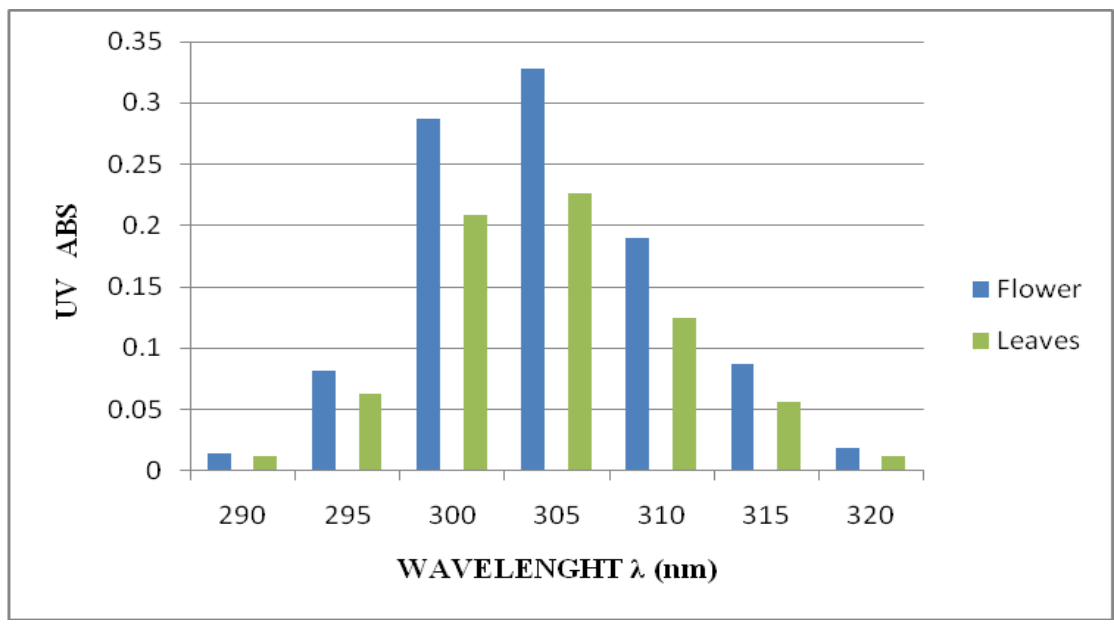

Fig. 1: UV radiation absorbance for T. patula Flower and Leaves at wavelength range $290-320 \mathrm{~nm}$

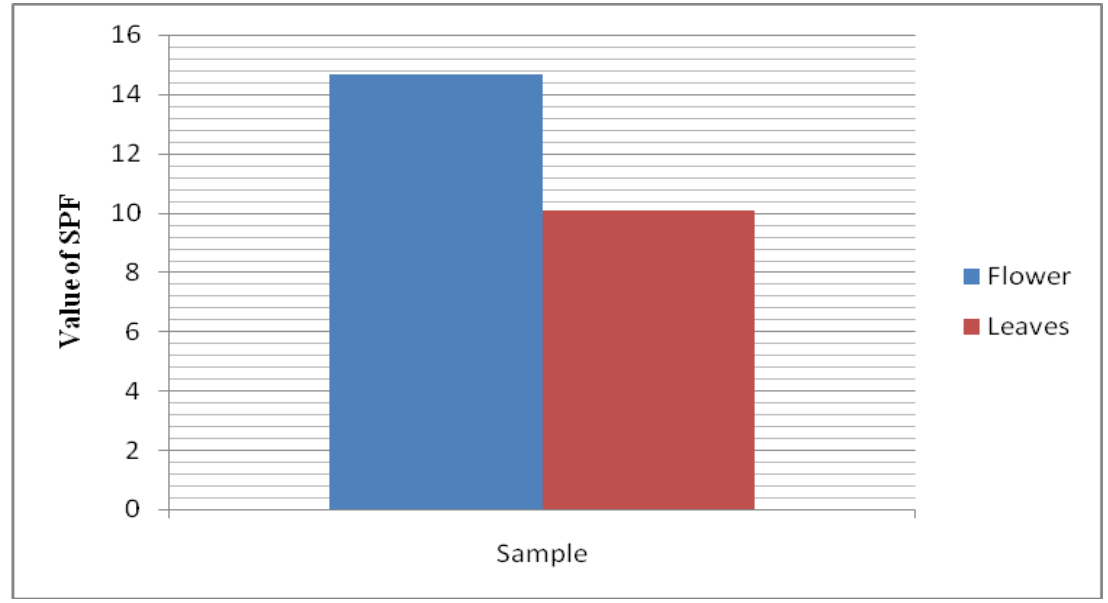

Fig. 2: Sun Protection Factor (SPF) for Tagetes patula Flower and Leaves

SPF factor significant value lies at $(P \geq 0.01)$ level; checked value was significant at $(P \geq 0.01)$ level. In this study, lesser absorption value of sun protection factor was observed in leaves as compare to flowers. In this respect, reflectance capacity of leaves is observed higher than flower by employing spectrophotometric methods. Spectrophotometrically SPF value of marigold flower and leaves is calculated and significantly value was obtained which is equivalent to 15 and 10 at $(P \geq 0.01)$ level. An evaluated SPF value showed the significant differences in SPF value of flower and leaves of marigold. Hence, developed cosmetic product from marigold containing SPF values in the range of $10-15$ considered to be safe for human with sensitive skin against directly exposed sunlight condition. This study supports photoactive in gradients of marigold which help reduces the harmful effects of UV irradiation on human skin.

\section{DISCUSSION}

The protection of human skin against the UV induces radiation sunscreen has used ${ }^{[12,13]}$. Plants having different types of active constituents, which worked as shield to protect the skin from UV rays ${ }^{[14]}$. Eco-friendly cosmetics are prepared with naturally extracted ingredients known as vegan cosmetics. These compounds are isolated from different parts of plants. These vegan cosmetics are a good source of different type of metabolites ${ }^{[15]}$ and antioxidant, having skin protection properties SPF ${ }^{[16]}$. In plants several types of secondary metabolites are presents such as ascorbic acid, catechins, triterpenes ${ }^{[16]}$, tannins, isoflavones, xanthophylls [17], flavones, coumarins, flavonoids, flavanones, phytoestrogens, anthocyanidines, carotenoids ${ }^{[18]}$, Volatile oils have been used as active compounds for sunscreens. These active compounds are also obtained from marine sources used as a preparation of anti-photo-ageing commercial products ${ }^{[19]}$. Different mechanisms are accountable for protection. UV rays scattered and reflected by inorganic substances alternatively organic molecules absorbed the radiation [20]. Cyclic or conjugated bonds and aromatic rings are dependable for the advancement of UV absorption 
profile ${ }^{[21]}$. The soluble polyphenols absorbed radiation from 304-350 nm while insoluble phenol from 352-385 $\mathrm{nm}{ }^{\text {[22] }}$. UVB radiation was absorbed by ferulic acid and its derivatives [23]. Two aromatic rings containing compounds and flavonoids absorbed the UV rays in the

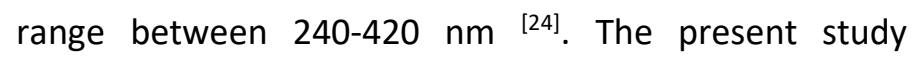
demonstrates that the marigold plants having SPF'S factors become a good source for the formulation of biocosmetics. This formulation was tested for long period and did not found any types of allergic reaction. Thus marigold extracts are suitable for use as natural sunscreen.

\section{CONCLUSIONS}

The SPF represents the effectiveness of a cosmetic product formulation specially sunscreens. The marigold should absorb the majority of UV radiation (290 to 320 $\mathrm{nm}$ ) so as to be effective in preventing skin cancer, wrinkle formation, photo ageing, sunburn and other skin damages. This study concluded that marigold plants are better alternative of cosmetic products to protect the skin from UV radiations. The method used in this work is simple, fast, not expensive and easy-to-use. Therefore, it could be used more often to monitories and evaluate the SPF value on sunscreens and other cosmetic products.

In future studies presence of secondary metabolites which are found in marigold plants must be good source for formulation of multifunctional sunscreen and other cosmetics, will not showed any adverse effect on skin.

\section{CONTRIBUTION OF AUTHORS}

Research concept- Deepshikha Kushwaha

Research design- Deepshikha Kushwaha

Supervision- Yashodhara Verma

Materials- Deepshikha Kushwaha

Data collection- Deepshikha Kushwaha

Data analysis and Interpretation- Deepshikha

Kushwaha and Prashant Katiyar

Literature search- Deepshikha Kushwaha

Writing article- Deepshikha Kushwaha

Critical review- Prashant Katiyar

Article editing- Deepshikha Kushwaha

Final approval- Yashodhara Verma

\section{REFERENCES}

[1] Ichihashi $M$, Ueda $M$, Budiyanto $A$, Bito $T$, Oka $M$, et al. UV-induced skin damage. Toxicol., 2003; 189: 2139.
[2] Mensah AY, Sampson J, Houghton PJ, Hylands PJ, Westbrook J, et al. Effects of Buddleja globosa leaf and its constituents relevant to wound healing. J Ethnopharmacol., 2001; 77: 216-21.

[3] Dutra EA, Oliveira DA, Kedor-Hackmann ERM, Santoro MI. Determination of sun protection factor (SPF) of sunscreens by ultraviolet spectrophotometry. Braz J Pharm Sci., 2004; 40: 38185.

[4] Detoni CB, Souto GD, Silva AL, Pohlmann AR, Guterres SS. Photostability and skin penetration of different E-resveratrol-loaded supramolecular structures. Photochem Photobiol., 2012; 88(4): 91321.

[5] Saewan N, Jimtaisong A. Photoprotection of natural flavonoids. J Appl Pharm Sci., 2013; 3(9): 129-41.

[6] Westerdahl J, Ingvar C, Masback A, Olsson H. Sunscreen use and malignant melanoma. Int. J. Cancer, 2000; 87: 145-50.

[7] Espinosa-Leal CA, Garcia-Lara S. Current methods for the discovery of new active ingredients from natural products for cosmeceutical applications. Planta Med., 2019; 85(7): 535-51.

[8] Farasat M, Khavari-Nejad RA, Baghernabavi SM, Namjooyan F. Antioxidant activity, total phenolic and flavonoid contents of some edible green sea weeds from northern coasts of the Persian gulf. Iran J Pharm Res., 2014; 13: 163-70.

[9] Mirshafa SA, Azadbakht M, Ahangar N. Study of antidipressant and sedative-hypnotic activity of hydroalcholic extract of Asperugo procumbens L. aerial parts in mice. Iran J Pharm Res., 2013; 12: 52935.

[10]Liu MC, Lin CT, Shau MD, Chen ZS, Chen MT. Studies on natural ultraviolet absorbers. J Food Drug Anal., 1996; 4: 243-48.

[11]Bonina F, Lanza M, Montenegro L, Puglisi C, Tomaino $A$, et al. Flavonoids as potential protective agents against photooxidative skin damage. Int J Pharm., 1996; 145: 87-91.

[12] Marronato A, Santos de Almeida T, Portugal-Mota J, Areias de Oliveira C, Rosado C, et al. Comparison of sunscreens containing titanium dioxide alone or in association with Cocoa, Murumuru Or Cupuaçu Butters. J Biomed Biopharm Res., 2016; 13(2): 22944. 
[13]Peres DA, Sarruf FD, de Oliveira CA, Velasco MVR, Baby AR. Ferulic acid photoprotective properties in association with UV filters: multifunctional sunscreen with improved SPF and UVA-PF. J. Photochem Photobiol B Biol., 2018; 185: 46-49.

[14]Moraes CAP, Areas EPG, Velasco MVR. Assessment of functional stability of photoprotective formulations containing rutin succinate. Cosmet., 2017;4(3): 27-28.

[15]Mansur MCPP, Leitao SG, Cerqueira-Coutinho C, et al. In vitro and in vivo evaluation of efficacy and safety of photoprotective formulations containing antioxidant extracts. Braz J Pharmacogn., 2016; 26(2): 251-58.

[16]Cavinato M, Waltenberger B, Baraldo G, Grade CVC, Stuppner $\mathrm{H}$, et al. Plant extracts and natural compounds used against UVB-induced photoaging. Biogeronto., 2017; 18(4): 499-516.

[17]Polonini HC, Brandao MAF, Raposo NRB. A natural broad-spectrum sunscreen formulated from the dried extract of Brazilian Lippia sericea as a single UV filter. RSC Adv., 2014; 4(107): 62566-75.

[18]Anunciato TP, da Rocha Filho PA. Carotenoids and polyphenols in nutricosmetics, nutraceuticals, and cosmeceuticals. J Cosmet Dermatol., 2012; 11: 51-54.
[19]Wang T, Jonsdottir R, Olafsdottir G. Total phenolic compounds, radical scavenging and metal chelation of extracts from Icelandic seaweeds. Food Chem., 2009; 116(1): 240-48.

[20]Pallela R, Na-Young Y, Kim SK. Anti-photoaging and photoprotective compounds derived from marine organisms. Mar Drugs, 2010; 8(4): 1189-202.

[21] Kim SK. Marine cosmeceuticals. J Cosmet Dermatol., 2014; 13: 56-67.

[22]Miksa S, Lutz D, Guy C, Delamour E. Sunscreen sun protection factor claim based on in vivo inter laboratory variability. Int J Cosmet Sci., 2016; 38(6): 541-49.

[23]Romani A, Campo M, Pinelli P. HPLC/DAD/ESI-MS analyses and anti-radical activity of hydrolyzable tannins from different vegetal species. Food Chem., 2012; 130(1): 214-21.

[24]Reis JS, Correa MA, Chung MC, Dos Santos JL. Synthesis, antioxidant and photoprotection activities of hybrid derivatives useful to prevent skin cancer. Bioorg Med Chem., 2014; 22(9): 2733-38. 